\title{
Construct of Metrology Information Public Service System
}

\author{
LI Yuanqinga, ${ }^{a}$, WU Shilin ${ }^{b}$, ZHANG qi \\ College of Mechatronic Engineering and Automation , National University of Defense Technology , \\ Changsha 410073,China,

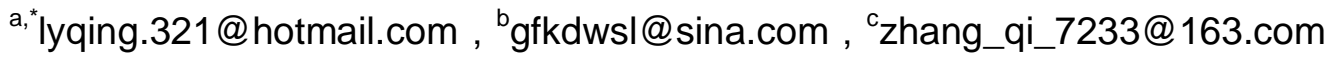

Keywords: Metrology, metrological support, management information system

\begin{abstract}
Industrial metrology systems for inspection and quality control are the key issues to ensure the high quality and consistency of metrology. However, the traditional metrology system contents multiple subsystem which separated by different functions and is hard to organized as a work flow in practice. In this paper, we reorganized the different metrology sub-system with the work flow in daily practice by using computer and information integration technology. In this metrology information public information service system, the work plan, instrument management, metrology regulations, auto-collaboration and efficiency assessment are combined together. By using this system, the higher quality and consistency of measurement can be confirmed.
\end{abstract}

\section{Introduction}

With the coming of information era and the rapid development of computer technique, metrology, one of the foundations of measuring technology, plays a more important role in technology innovation, high-tech industry and even the economy development[1]. On the other hand, the development of metrology itself also be supported by computer and information technique, in other words, metrological should take advantage of computer technique to improve itself.

\section{Ways to Improve Metrology Business}

Standardization. Metrology business covers all aspects of measuring and calibration process, like instruments management, personnel management, task allocation, data processing, and it usually contains lots of procedure. Thus, to simplify the business process of metrology under the premise that does not affect the reliability, consistency and traceability is quite necessary.

Automation. Instrument science and technology is continuing to improve in recent years, so that most of the measuring instrument is equipped with general interface port for hardware and software in case of reading and writing data directly by computers. That provides a convenient way of automate measuring and calibrating process to using computers instead of manual operation. 
Automation not only greatly reduces the time and cost, but also regulates the metrology procedures, and make the calibration process strictly accordance with the metrological regulations.

Database. Metrology is more and more widely used in production, scientific research, economy and other fields with its gradually reduced cost, accordingly metrological activities become more frequent. In addition, it's possible to collect data quickly at any time from instrument (even real-time collection) at present. Subsequently, there is a massive increase in metrology data, which brings about a mountain of trouble. Therefore, establishing large database to store, query, and analyze massive data in metrology business is just around the corner.

\section{Weakness of Industrial System}

As far as we know, metrology management system, also named as metrology task management software, is developed and applied in several institutions and it largely improve the efficiency of the metrological work by making efforts to standardize metrological procedure. However, the industrial software and system still have following problems.

Scheduling Plays the Main Role. Generally, a metrology management system consists of personnel management, instruments management, and business management for certain institutions[2,4]. Institutions apply this system to calibration and verification works by assigning missions to different departments and let them cooperate to accomplish metrology works. As you can see, the scheduling of instruments and personnel allocation is the main function. In other words, the metrology management system is similar to $\mathrm{MRP}$ (material resource planning) in a company[6], which gives priority to the material, as the instruments for metrology, and that is dominated by metrology business schedule.

Limited in Metrology Support. The metrology management system now in use has certain limitations in metrology support, and that makes less use in calibration, data processing, and other metrological activities, while these activities are the most significant part of metrology and measurement.

Data Redundancy. Calibration and verification produce large amounts of data, and some of them contain information while some of them are redundant. Industrial software or system usually do achieve, store and query works of data, but are not able to discriminate and eliminate bad data, not mentioning optimization of data, or providing statistics accordingly with the behavior of metrological works at all[3]. 


\section{Construct of Metrology Information Public Service System}

Combined with the shortage of industrial metrology management software under, and the condition of information era, as strengthen the comprehensive integration of all aspects of metrological resources, we construct a metrology information public service system which aims to improve the level of metrological technique.

Function Modules. System contains 6 foundational elements of modern metrology, including metrology resources module, work plan module, metrology support and statistics module, verification and calibration module, data processing and analysis module, system maintenance and management module. Modules are inner associated, and each of the modules is separated in function but not in physical. Fig 1 is the view of function module in details that each rectangular means a function module and the arrow point to its sub-modules.

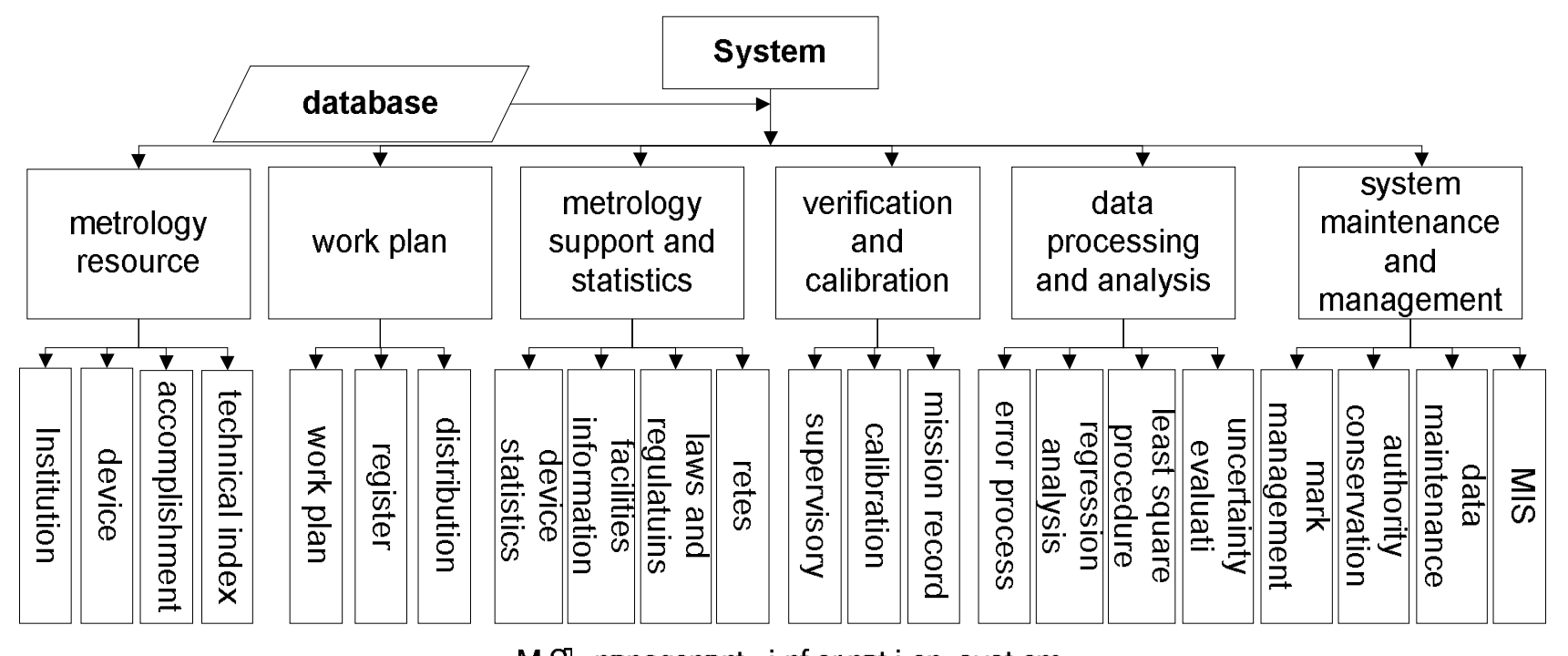

MSI management i nf or nat i on syst em

Figure 1 Function Module

Improvements. System broadens the wide in several aspects, and is attached to several features on the basis of industrial metrology management software.

(1) Regulations

Technical regulations are the key to ensuring technique normative, reliable, consistent and traceable, which are the four foundational features of metrology. Regulations contained in the system mainly include the national standards, the specifications of calibration, the procedures of verification, and the metering work files. The system automatic load these regulations integrated the metrological work procedures, and which is convenient of users consulting, invoking, downloading and referencing the regulations.

(2) Effectiveness Evaluation 
Effectiveness evaluation is a creative function of the system and also is of significance in public services. The evaluation files indicate the status of instruments, organization, personnel, regulations and other basic elements of the metrology, and furthermore show statistics such as instrument coverage, examination rate, which could be demonstrated visually in figure and tables. That mainly includes the following aspects:

(1) instrument statistics: the sum, allocation and index information of instruments such as standard metrology device, calibration device, standard substance and so on;

(2) institution statistics: the name, location and strength level of each institution, which allow system users to compare and choose appropriate institution to accomplish their metrology work;

(3) metrology personnel statistics: such as the sum of staff, title information, major distribution, and so on;

(4) comprehensive statistics: the data after processing and analysis, such as instruments' coverage, examination rate, vacancy rate and so on.

(3) Data Processing

System allows users to input data, do error processing and data analysis, which makes it convenient of users to get accurate results just in time. Specifically, data processing module includes gross error rejecting, system error and random error processing, least square fitting and regression analysis, and also contains calculation and auxiliary calculation of measurement uncertainty, combined standard uncertainty and expanded uncertainty. These capabilities are perfectly suited for metrological work in practice.

Implementation. The key of the metrology information public service systems' implementation is comprehensive. Since the system covers a wide range of metrology and go deep into the all aspects of metrological works, combination and integration of industrial software and newly program is critical. By using Visual Studio 2010 web application and on basis of .NET Framework 3.5 we write a program, on which uses can log in as client through network.

Specifically, there are three layer of the system. The first layer is the server, including the main structure of the integrated service system, and is the top layer. Its input and output is system stream that have the highest authority. Database is attached to this layer, and server could give authority to other layers which permit them query and update data items, but the tables of the database could be only modified by server's command. The second layer is the middle layer, which is made up of clients. Each client connection with the server through network in certification, and its authority fixed at a time. Metrological personnel mainly work in this layer. They update instruments, personnel, and institution information into the database, and meanwhile they do data processing by means of the clients' program. The third layer is the underlying consisting of a variety of 
instruments. In this layer calibration and verification practice and row data are generated. The specific physical hierarchy view is shown in Fig 2.

Clients, in the second layer, get data through auto-calibration software or manual input records. In the realization of auto-calibration, we embed several soft wares into the system, such as METCAL from Fluke and custom-made LabView program, within thousands of calibration manuals and procedures. For good running of public service system, system is open to all kinds of general hardware and software port. Besides, all these software must input their metrological into database regularly and consistently to make sure that the whole system always keep its reliability and security.

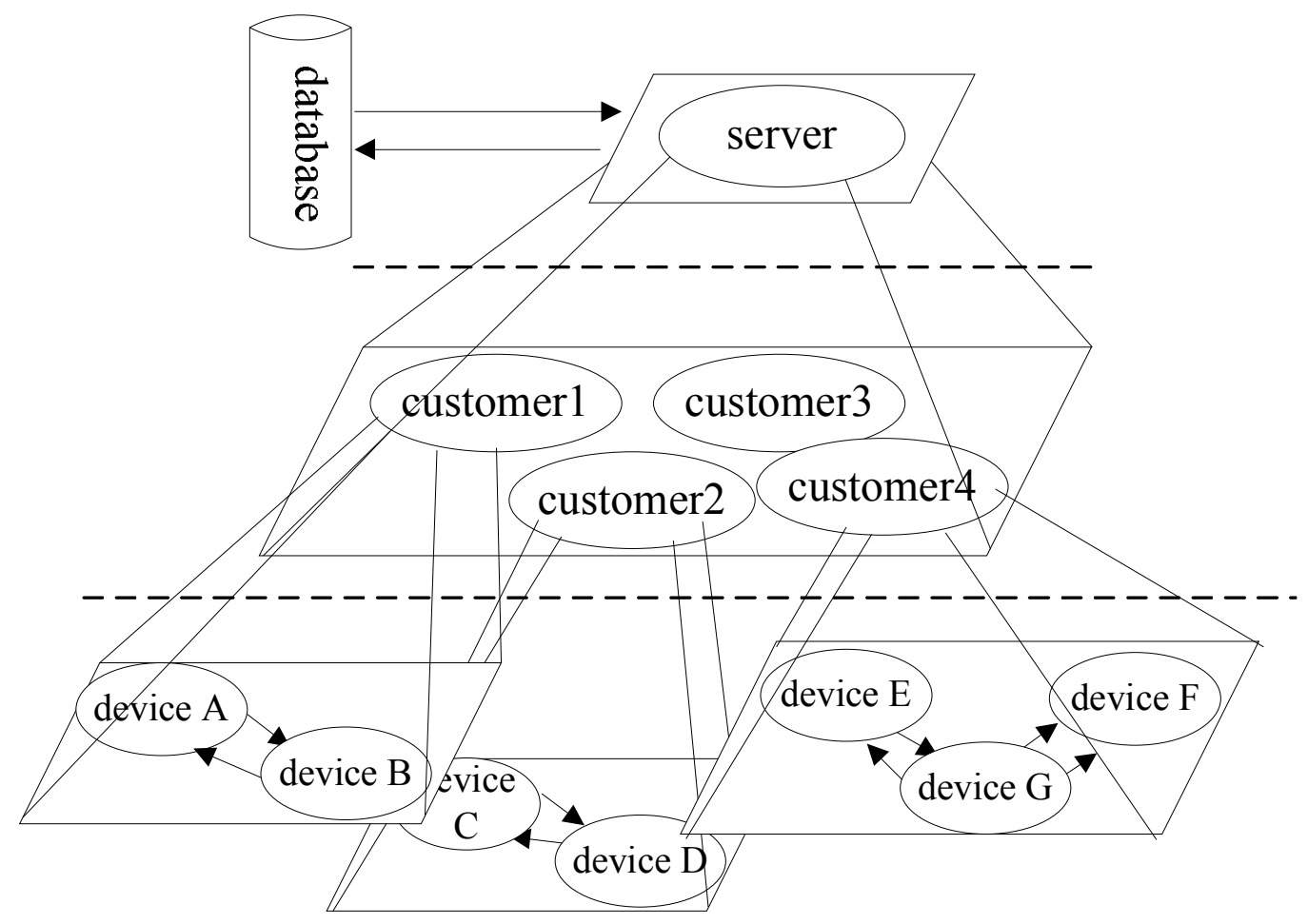

Figure 2 Physical Hierarchy

\section{Summary}

Varied industrial metrology systems are in use currently, and they are developed and applied in different organizations and institutions separately and individually. Metrology Information Public Service System is a higher system functioning like the ERP (enterprise resource planning) of the enterprise, which jumps out of the traditional task management software's boundaries, broadens the scope for metrology management from scheduling to optimizing metrological resources.

Still and all, the system needs to be improved and perfected in the following aspects.

(1)Strengthen security guarantee by tightening permission and certificate, and reasonable divide jurisdiction of client from one another. 
(2)Expand application scope by inviting regional and national metrology institute to join the public service system, thus could make full use of system's function and make it run at maximum efficiency.

(3)Strengthen the integration and combination of each module by removing excess units, and make the system operate more convenient and perform more friendly.

\section{References}

[1] Zhang qi, Wu Shilin, Ouyang Hongjun, Xiong Jiulong, Military Metrology Foundation, first ed, National University of Defense Technology Press, Changsha, 2013

[2] Dang Yanhu, Collaborative system design and implementation based on the calibration of measurement, J, Information Technology and Control. 23(2013) 28-30

[3] Wang Xin, Guo Meixia, The analysis and countermeasures of Metrology Information Management System's operating situation, J, Computer Technology and Application. 32(2012) $58-60$

[4] Ding Tao, Xu Erqiang, Liu Zhong, Shao Huailing, Production scheduling system design of metrology center at provincial level, J, Electric Power System. 5(2012) 34-37

[5] Zhang Weiyan, Wang Xinlin, Brief introduction of metrology management software in the application of measuring institution, J, Information Science and Technology. 12(2013) 245-246

[6] Li Dong, The Theory and Application of Management Information System, third ed, Peking University Press, Beijing, 2007

[7] Zheng Renjie, Yin renkun, Tao Yonglei, Practical Software Engineering, second ed, Tsinghua University Press, Beijing, 2008 